\title{
Differential rotation and meridional flows in stellar convection zones
}

\author{
Manfred Küker and Günther Rüdiger \\ Leibniz-Institut für Astrophysik Potsdam \\ An der Sternwarte 16, 14482 Potsdam, Germany \\ email: mkueker@aip.de, gruediger@aip.de
}

\begin{abstract}
Differential rotation and meridional flow are key ingredients in flux transport dynamo models of the solar activity cycle. As the subsurface flow pattern is not sufficiently constrained by observations, it is a major source of uncertainty in solar and stellar dynamo models. We discuss the current mean field theory of stellar differential rotation and meridional flows and its predicitons for the Sun and stars on the lower main sequence.
\end{abstract}

Keywords. Sun: activity, Stars: activity, Sun: rotation, Stars: rotation

\section{Introduction}

Differential rotation is the main field generator in both the $\alpha-\Omega$ dynamo and the flux transport dynamo. In the flux transport dynamo, which is currently the most favored model, the meridional flow determines the cycle period. While the internal rotation of the solar convection zone is known from helioseismology, the meridional flow has so far been measured reliably at the surface, where it is directed towards the poles. Mass conservation requires a return flow towards the equator but the depth at which that occurs is not known. While Schad et al. 2012 did not find a return flow for radii down to $0.8 R_{\odot}$, Zhao et al. report a reverse flow direction between $0.91 R_{\odot}$ and $0.82 R_{\odot}$.

For stars even the surface differential rotation has to be inferred from observations rather than measured directly and the meridional flow is unknown. The combined data from various methods methods suggests that the surface differential rotation of main sequence stars is only weakly dependent on the stellar rotation rate but strongly depends on the effective temperature. Barnes et al. (2005) presented a power law fit of the form $\delta \Omega \propto T^{8.92}$ for the temperature dependence of the surface differential rotation which was supported by the findings of Reiners (2006).

\section{Mean field theory: predictions for low mass stars}

Mean field theory uses averages to describe the large-scale behavior of the gas in the convection zones of the Sun and other low-mass main sequence stars. The model described in Küker et al. (2011) solves the equations of angular momentum transport, meridional flow, and convective heat transport using transport coefficients derived with the second order correlation approximation and model convection zones computed with the MESA stellar evolution code (Paxton et al. 2011.)

Our model predicts solar-type differential rotation in all cases and meridional flow with one flow cell per hemisphere. The surface flow is directed towards the poles and the return flow towards the equator is located at the bottom of the convection zone. The left panel in Figure 1 shows the surface differential rotation, $\delta \Omega=\Omega_{\max }-\Omega_{\mathrm{min}}$, predicted by the model for a sequence of ZAMS stars rotating with a period of $2.5 \mathrm{~d}$, the power law fit of 

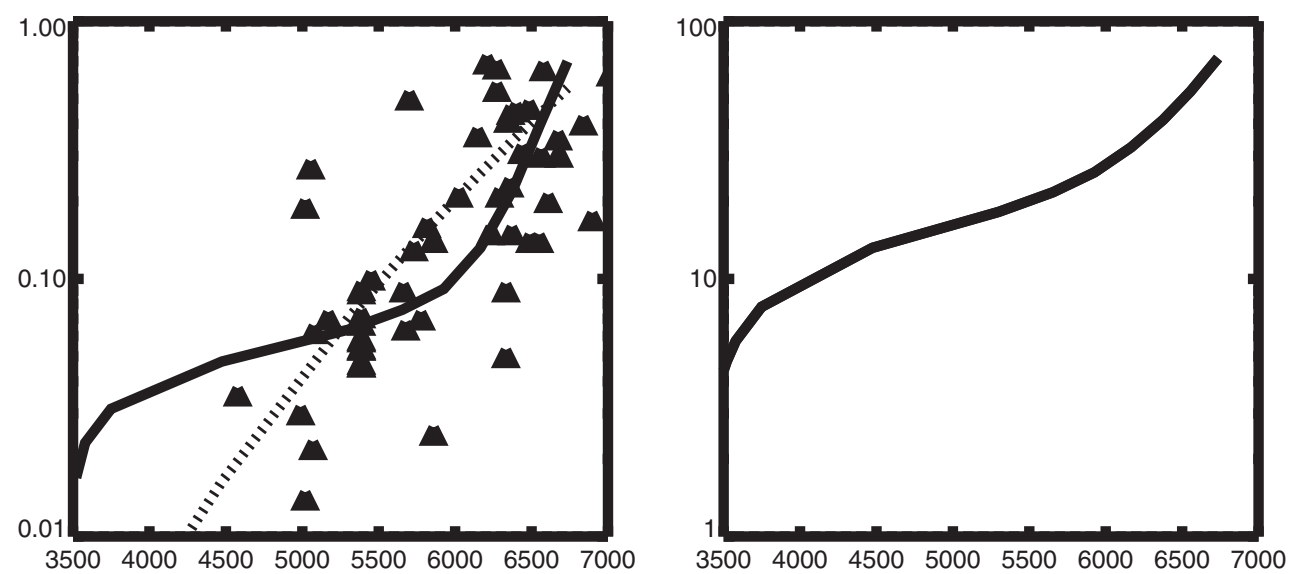

Figure 1. Left:Surface differential rotation in rad/day vs. effective temperature in K. The solid line indicates the prediction of the mean field model for zero age main sequence stars rotating with a period of $2.5 \mathrm{~d}$. The dotted line indicates the power law found by Barnes et al. (2005). The triangles represent surface differential rotation values of derived from observations. Right: Surface meridional flow in $\mathrm{m} / \mathrm{s}$ vs. effective temperature in $\mathrm{K}$.

Barnes et al. (2005), and the observed differential rotation values as compiled in Reiners (2006). The mean field model predicts a temperature dependence that is quite different from the power law. The curve is much flatter than the power law for temperatures below $6000 \mathrm{~K}$ and steeper for temperatures above that value. While Barnes et al. list several lower main sequence stars with differential rotation much weaker than predicted by the mean field model, the latter is strongly supported by data recorded by the Kepler spacecraft (Reinhold \& Reiners 2013).

The right part of Figure 1 shows the surface meridional flow vs. the effective temperature corresponding to the differential rotation shown in the left part. Like the differential rotation, the meridional flow increases with temperature and the increase is steeper for temperatures above $6000 \mathrm{~K}$ than below. The steepening if the curve is less pronounced though. Assuming a flux transport dynamo for all main sequence stars in the temperature range shown we would therefore expect a the cycle period to decrease with increasing temperature, i.e. F stars should have cycle periods substantially shorter than the 11 year solar cycle.

\section{References}

Schad, A., Timmer, J., \& Roth, M. 2012, AN 333, 991

Zhao, J., Bogart, R. S., Kosovichev, A. G., Duvall, T. L. Jr., \& Hartlepp, Th. 2013, ApJL, 774, L29

Barnes, J. R., Collier Cameron, A., Donati, J.-F., James, D. J., Marsden, S. C., \& Petit, P. 2005, MNRAS, 357, L1

Reiners, A. 2006, A\&A, 446, 267

Küker, M., Rüdiger, G., \& Kitchatinov, L. L. 2011, A\&A, 530, A48

Paxton, B., Bildsten, L., Dotter, A., Herwig, F., Lesaffre, P., \& Timmes, F. 2011, ApJL, 192, 3

Reinhold, T. \& Reiners, A. 2013, A \& A 557, A11 